\title{
Внутризонное поглощение излучения дырками в квантовых ямах InAsSb/AISb и InGaAsP/InP
}

\author{
(C) Н.В. Павлов ${ }^{1}$, Г.Г. Зегря ${ }^{1,2}$, А.Г. Зегря ${ }^{1}$, В.Е. Бугров ${ }^{2}$ \\ ${ }^{1}$ Физико-технический институт им. А.Ф. Иоффе Российской академии наук, \\ 194021 Санкт-Петербург, Россия \\ ${ }^{2}$ Университет информационных технологий, механики и оптики (ИТМО), \\ 197101 Санкт-Петербург, Россия \\ E-mail: pavlovnv@mail.ru
}

(Получена 11 мая 2017 г. Принята к печати 30 июня 2017 г.)

В рамках четырехзонной модели Кейна выполнен микроскопический анализ механизма внутризонного поглощения излучения дырками с переходом их в спин-отщепленную зону для квантовых ям на основе полупроводников InAsSb/AlSb и InGaAsP/InP. Расчет проведен для двух поляризаций падающего излучения: вдоль оси роста кристалла и в плоскости квантовой ямы. Показано, что поглощение с переходом в дискретный спектр спин-отщепленных дырок имеет бо́льшую интенсивность, чем для переходов в непрерывный спектр. Проведен подробный анализ зависимости коэффициента внутризонного поглощения от температуры, концентрации дырок и ширины квантовой ямы. Показано, что данный процесс может быть основным механизмом внутренних потерь излучения для лазеров на квантовых ямах.

DOI: 10.21883/FTP.2018.02.45445.8645

\section{1. Введение}

В лазерных структурах на основе узкощелевых полупроводников (лазеры ближнего и среднего инфракрасного диапазона) при высоких температурах наблюдается сильное внутризонное поглощение излучения, которое ведет к срыву генерации $[1,2]$. С уменьшением толщины активной области внутризонное поглощение возрастает, и в лазерных структурах с квантовыми ямами коэффициент поглощения может достигать величины в несколько десятков $\mathrm{cm}^{-1}$.

Механизмы внутризонного поглощения излучения в лазерах на квантовых ямах (КЯ) исследуются как теоретически, так и экспериментально на протяжении многих лет [3]. Экспериментальные результаты показывают, что коэффициент внутризонного поглощения лазерного излучения в несколько раз выше, чем предсказывает теория. Ранее в работе [4] изучено внутризонное поглощение света в квантовых ямах, вызванное различием в эффективных массах электронов в квантовой яме и в гетеробарьере. Оптические переходы между локализованными состояниями в квантовой яме исследованы также в [5]. В работе [6] была решена задача о внутризонном поглощении излучения дырками в квантовых ямах в рамках модели Латтинжера. В работе [7] был проведен расчет коэффициента поглощения излучения дырками с переходом в спин-отщепленную (so) зону в рамках модификации метода огибающих, предложенной в работе [8]. Однако данный метод предполагает использование численного моделирования с самого начала расчетов и не позволяет провести подробный анализ полученных результатов в зависимости от параметров гетероструктуры. В данной работе мы будем использовать четырехзонную модель Кейна [9], которая также основана на использовании $\mathbf{k P}$ гамильтониана $8 \times 8$, однако позволяет практически без потери точности получить явные аналитические выражения для энергетических спектров и волновых функций носителей заряда, а также матричных элементов переходов.

Цель работы состоит в исследовании внутризонного поглощения излучения дырками с переходом их в спинорбитально отщепленную зону для квантовых ям на основе полупроводников $\mathrm{A}^{\mathrm{III}} \mathrm{B}^{\mathrm{V}}$. Расчет коэффициента поглощения выполнен для двух поляризаций электромагнитной волны: перпендикулярной и параллельной гетерогранице. В работе выполнен подробный анализ зависимости коэффициента поглощения от температуры, концентрации дырок и ширины квантовой ямы. Также показано, что механизм внутризонного поглощения дырками является более эффективным, чем электронами [10]. Расчеты выполнены на примере гетероструктуры с глубокой квантовой ямой $\mathrm{AlSb} / \mathrm{InAs}_{0.84} \mathrm{Sb}_{0.16} / \mathrm{AlSb}$ (рис. $1, a)$, которая является одной из наиболее перспективных структур для создания полупроводникового лазера среднего инфракрасного диапазона, работающего при комнатной температуре [11-13], а также гетероструктуры InGaAsP/InP (рис. 1, $b$ ), которая используется в конструкциях полупроводниковых лазеров с длиной волны излучения 1.55 мкм [14].

\section{2. Дырки в однородном полупроводнике}

Для нахождения скорости внутризонного поглощения необходимо иметь волновые функции носителей заряда, которые в случае узкощелевых полупроводников необходимо вычислять в многозонном приближении. Здесь будет использована четырехзонная модель Кейна [9], которая позволяет с достаточной степенью точности получить аналитические выражения для энергетического спектра и волновых функций носителей заряда в по- 

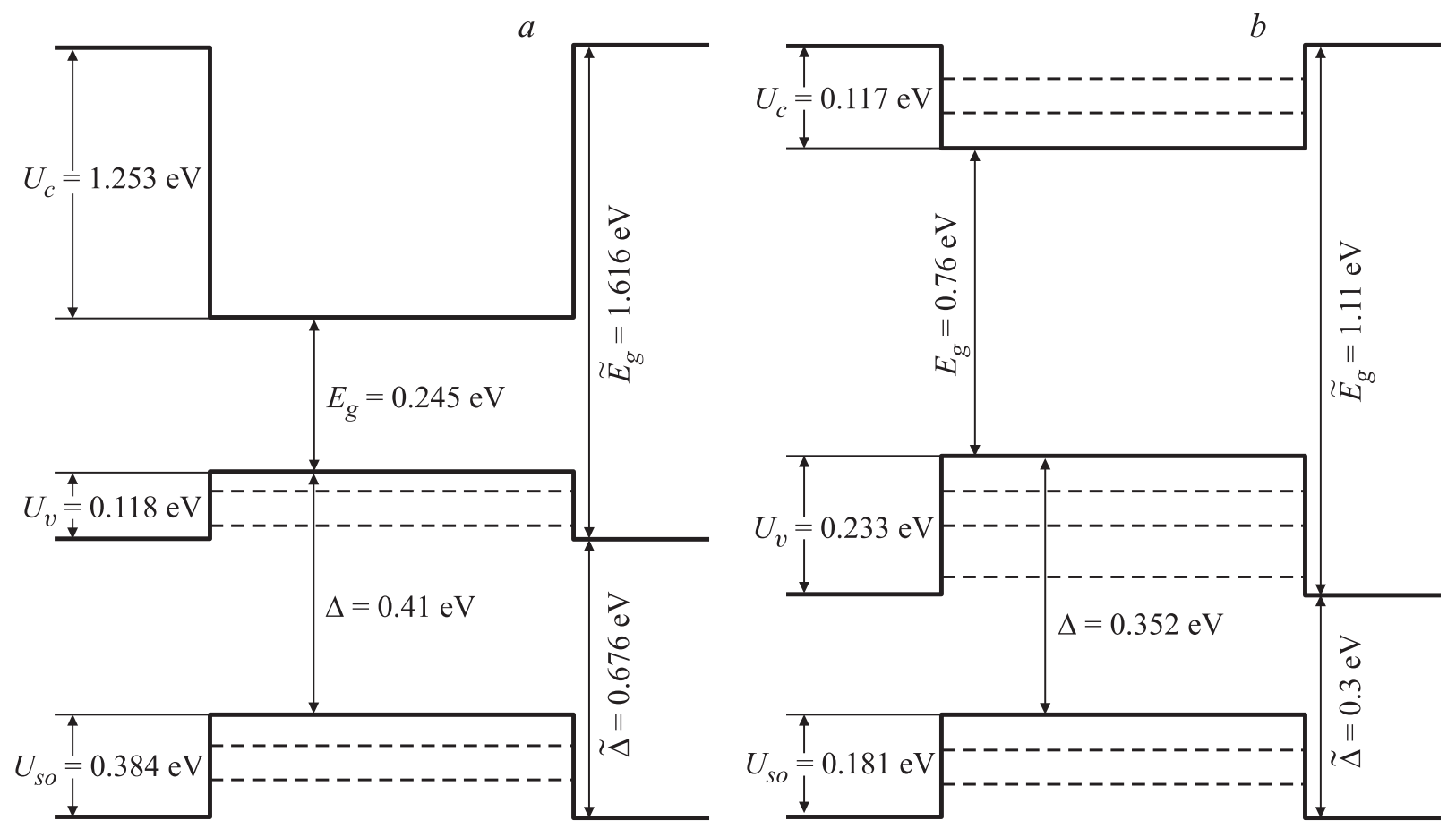

Рис. 1. Зонные диаграммы гетероструктуры с глубокой квантовой ямой $\mathrm{AlSb} / \mathrm{InAs}_{0.84} \mathrm{Sb}_{0.16} / \mathrm{AlSb}$ (a) и гетероструктуры $\operatorname{InGaAsP/InP~}(b)$. Штриховые линии - уровни размерного квантования тяжелых и спин-отщепленных дырок.

лупроводниковых соединениях $\mathrm{A}^{\mathrm{III}} \mathrm{B}^{\mathrm{V}}$. Для полупроводников $\mathrm{A}^{\mathrm{III}} \mathrm{B}^{\mathrm{V}}$ волновые функции зоны проводимости в центре зоны Бриллюэна описываются представлением $\Gamma_{6}^{+}$, а валентной зоны - представлениями $\Gamma_{7}^{+}$и $\Gamma_{8}^{+}$. Два первых из них дважды вырождены, а последнее четырехкратно вырождено. Уравнения для соответствующих волновых функций могут быть записаны в дифференциальной форме. Для наших целей подходящим является следующее представление базисных волновых функций:

$$
|s \uparrow\rangle,|s \downarrow\rangle,|x \uparrow\rangle,|x \downarrow\rangle,|y \uparrow\rangle,|y \downarrow\rangle,|z \uparrow\rangle,|z \downarrow\rangle,
$$

где спиноры $|s\rangle$ и $|x\rangle,|y\rangle,|z\rangle$ - блоховские функции $s$ - и $p$-типа с угловым моментом 0 и 1 соответственно; $s$-функции описывают состояние зоны проводимости, а $p$-функции - состояние валентной зоны в Г-точке; стрелкой обозначено направление спина. Волновая функция носителей заряда $\psi$ может быть представлена в виде [15]

$$
\psi=\Psi|\mathbf{u}\rangle=\Psi_{s}|s\rangle+\Psi_{p}|\mathbf{p}\rangle,
$$

где $\boldsymbol{\Psi}, \boldsymbol{\Psi}_{s}, \boldsymbol{\Psi}_{p}-$ спиноры. Вблизи Г-точки уравнения для огибающих $\Psi_{s}$ и $\boldsymbol{\Psi}_{p}$ в сферическом приближении имеют следующий вид $[16,17]$ :

$$
\begin{gathered}
\left(E_{c}-E\right) \Psi_{s}-i \hbar \gamma \nabla \Psi_{p}=0 \\
\left(E_{v}-\delta-E\right) \Psi_{p}-i \hbar \gamma \nabla \Psi_{s}+\frac{\hbar^{2}}{2 m_{0}}\left(\gamma_{1}+4 \gamma_{2}\right) \nabla\left(\nabla \Psi_{p}\right) \\
-\frac{\hbar^{2}}{2 m_{0}}\left(\gamma_{1}-2 \gamma_{2}\right)\left[\nabla\left[\nabla \Psi_{p}\right]\right]+i \delta\left[\sigma \Psi_{p}\right]=0
\end{gathered}
$$

Здесь $\gamma-$ кейновский матричный элемент, имеющий размерность скорости, $\gamma_{1}$ и $\gamma_{2}=\gamma_{3}$ - обобщенные параметры Латтинжера, $\delta=\Delta / 3, \Delta-$ константа спинорбитального расщепления, $E_{c}$ и $E_{v}$ - энергии нижнего края зоны проводимости и верхнего края валентной зоны соответственно, $m_{0}$ - масса свободного электрона, $\sigma=\left(\sigma_{x}, \sigma_{y}, \sigma_{z}\right)-$ матрицы Паули. В первом уравнении системы (2) опущено слагаемое с тяжелой массой для электронов.

Из (2) следуют законы дисперсии дырок в однородном полупроводнике. В частности, для тяжелых дырок

$$
E=\delta-\frac{\hbar^{2} K_{h}^{2}}{2 m_{h}}
$$

где $m_{h}$ - масса тяжелой дырки. Энергия верхнего края валентной зоны $E_{V}$ для удобства положена равной $\delta$.

Законы дисперсии легких и спин-отщепленных дырок даются общей формулой

$$
\begin{aligned}
K^{2}= & -\frac{m_{l}+m_{h}}{\hbar^{2}} E \\
& -\frac{m_{h}}{\hbar^{2}} \delta \pm \sqrt{\left(\frac{m_{h}-m_{l}}{\hbar^{2}} E+\frac{m_{h}}{\hbar^{2}} \delta\right)^{2}+\frac{8 m_{h} m_{l} \delta^{2}}{\hbar^{4}}} .
\end{aligned}
$$

Выражение с положительным знаком соответствует легким дыркам, а с отрицательным - спин-отщепленным дыркам.

В (4) входит зависящая от энергии величина $m_{l}$ :

$$
m_{l}^{-1}=\frac{2 \gamma^{2}}{E_{g}+\delta-E}+m_{0}^{-1}\left(\gamma_{1}+4 \gamma_{2}\right) .
$$

Здесь $E_{g}=E_{c}-E_{v}$ - ширина запрещенной зоны полупроводника. В случае равенства нулю константы спин- 
орбитального взаимодействия $m_{l}$ совпадает с эффективной массой легкой дырки.

В Г-точке $(K=0)$ выражение (4) дает для ветви легких дырок $E_{1}=\delta$, а для ветви спин-отщепленных дырок $E_{2}=-2 \delta$. В окрестности Г-точки энергии $E_{1,2}$ могут быть разложены в ряд по волновому вектору:

$$
E_{1}=\delta-\frac{\hbar^{2} K^{2}}{2 m_{l h}}, \quad E_{2}=-2 \delta-\frac{\hbar^{2} K^{2}}{2 m_{s o}},
$$

где $m_{l h}, m_{s o}$ - эффективные массы легких и $s o$-дырок. Параболические спектры (6) часто используются в литературе.

\section{3. Волновые функции и спектр дырок в прямоугольной квантовой яме}

\section{1. Симметрия волновой функции}

Рассмотрим прямоугольную квантовую яму, расположенную следующим образом: ось $x$ направлена перпендикулярно гетерограницам, а область квантовой ямы лежит в пределах от $-a / 2$ до $a / 2$, где $a-$ ширина квантовой ямы. Квантовая яма симметрична относительно плоскости $y z$, что приводит к двукратному вырождению энергетических уровней. Для нумерации состояний с одинаковой энергией мы будем использовать то же квантовое число $v= \pm 1$, что и авторы $[16,17]$. Ортогональные друг другу волновые функции, отвечающие одной и той же энергии, должны удовлетворять уравнениям

$$
\begin{aligned}
& \Psi_{s \uparrow}(x, y, z)= \pm \Psi_{s \downarrow}(-x, y, z), \\
& \Psi_{x \uparrow}(x, y, z)=\mp \Psi_{x \downarrow}(-x, y, z), \\
& \Psi_{y \uparrow}(x, y, z)= \pm \Psi_{y \downarrow}(-x, y, z), \\
& \Psi_{z \uparrow}(x, y, z)=\mp \Psi_{z \downarrow}(-x, y, z) .
\end{aligned}
$$

Квантовому числу $v=1$ соответствуют верхние знаки, а $v=-1-$ нижние. Волновые функции с различными $v$ обладают разной симметрией.

\section{2. Граничные условия}

В литературе обсуждаются несколько приближенных методов получения граничных условий для волновых функций носителей в квантовой яме. Используемые нами граничные условия являются следствием метода, развитого Бертом [18], и закона сохранения потока вероятности $[16,17]$. Наиболее простой вид граничные условия принимают в случае использования приближений $\gamma=$ const, $\gamma_{1}=$ const и $\gamma_{2}=$ const. В этом случае $m_{h}=$ const. Также учитывается, что $m_{h} \gg m_{l}[16,17]$. В результате граничные условия представляют собой условия непрерывности на интерфейсе величин $\Psi$ и $\partial \Psi_{x} / \partial x$.

В широкозонной области величину запрещенной зоны будем обозначать $\tilde{E}_{g}$, а константу спин-орбитального взаимодействия $\tilde{\Delta}$. Связь $\tilde{E}_{g}$ с $E_{g}$ дается выражением $\tilde{E}_{g}=E_{g}+U_{c}+U_{v}$, где $U_{v}$ - глубина квантовой ямы для дырок, а $U_{c}$ - глубина квантовой ямы для электронов. Глубина квантовой ямы для спин-отщепленных дырок составляет $U_{s o}=U_{v}+\tilde{\Delta}-\Delta$.

Энергию $E$ в квантовой яме удобно отсчитывать от уровня, находящегося на $\delta$ ниже потолка валентной зоны. Иногда мы будем использовать еще и дополнительную ось энергии $\tilde{E}$, началом отсчета которой положим уровень, находящийся на $\tilde{\delta}$ ниже потолка валентной зоны широкозонной области $E_{v}^{B}=\delta-U_{v}$. Значения $\tilde{E}$ и $E$ связаны уравнением $\tilde{E}=E+U_{v}+\tilde{\delta}-\delta$.

\section{3. Волновые функции связанных so-дырок}

Волновые функции тяжелых дырок подробно исследованы в [16,17], и мы их приводим в Приложсении. Выпишем в явном виде огибающие волновых функций $s o$-дырок (в дальнейшем для простоты вместо слов „огибающие волновых функций“ мы будем писать „волновые функции“). Подмешиванием легких и тяжелых дырок к спин-отщепленным мы будем пренебрегать, так как при энергиях, меньших $-2 \delta$, компоненты волновых функций легких и тяжелых дырок быстро осциллируют и вклад от них в интегралы перекрытия ничтожно мал. Как следует из граничных условий, в этом случае компоненты $\Psi_{s}$ и $\Psi_{p}$ для ветви $s o$-дырок должны считаться непрерывными. При пренебрежении быстро осциллирующими вкладами подзон легких и тяжелых дырок спектр so-дырок может быть как непрерывным, так и дискретным [16,17]. Выберем систему координат так, чтобы продольная компонента волнового вектора совпала с осью $y$. Волновые функции связанных so-дырок в области квантовой ямы с $v=1$ будем искать в виде

$$
\begin{gathered}
\Psi(x)=D_{1}\left(\begin{array}{c}
i S_{d} \cos k x \eta \\
k \sin k x \eta-\lambda q \cos k x \xi \\
-i q \cos k x \eta+i \lambda k \sin k x \xi \\
-\lambda k \sin k x \xi+\lambda q \cos k x \eta
\end{array}\right) \\
+D_{2}\left(\begin{array}{c}
i S_{d} \sin k x \xi \\
-k \cos k x \xi-\lambda q \sin k x \eta \\
-i \lambda k \cos k x \eta-i q \sin k x \xi \\
-\lambda q \sin k x \xi-\lambda k \cos k x \eta
\end{array}\right) .
\end{gathered}
$$

Здесь $k$ и $q-x$ - и $y$-компоненты квазиимпульса спинотщепленных дырок, $S_{d}=\hbar \gamma\left(k^{2}+q^{2}\right) /\left(E_{g}+\delta-E\right)-$ амплитуда $s$-компоненты волновой функции в квантовой яме,

$$
\lambda=\frac{\delta}{E+\delta+\hbar^{2}\left(k^{2}+q^{2}\right) / 2 m_{h}}
$$

- параметр, зависящий от энергии, $D_{1}$ и $D_{2}$ - нормировочные коэффициенты,

$$
\xi=\frac{1}{\sqrt{2}}\left(\begin{array}{c}
1 \\
-1
\end{array}\right), \quad \eta=\frac{1}{\sqrt{2}}\left(\begin{array}{l}
1 \\
1
\end{array}\right) .
$$

Здесь и далее мы опускаем в выражениях для волновых функций дырок множитель $\exp ($ iqy). Именно этот 
множитель обеспечивает непрерывность $q$ на гетерограницах, а также сохранение $q$ при переходах между различными состояниями.

В области барьера, $x>a / 2$, волновые функции связанных дырок экспоненциально затухают при удалении от интерфейса:

$$
\begin{gathered}
\Psi(x)=\tilde{D}_{1}\left(\begin{array}{c}
i \tilde{S}_{d} \eta \\
\kappa \eta-\tilde{\lambda} q \xi \\
-i q \eta+i \tilde{\lambda} \kappa \xi \\
-\tilde{\lambda} \kappa \xi+\tilde{\lambda} q \eta
\end{array}\right) e^{-\kappa(x-a / 2)} \\
+\tilde{D}_{2}\left(\begin{array}{c}
i \tilde{S}_{d} \xi \\
\kappa \xi-\tilde{\lambda} q \eta \\
-i \tilde{\lambda} \kappa \eta-i q \xi \\
-\tilde{\lambda} q \xi+\tilde{\lambda} \kappa \eta
\end{array}\right) e^{-\kappa(x-a / 2)} .
\end{gathered}
$$

Здесь

$$
\tilde{S}_{d}=\frac{\hbar \gamma\left(-\kappa^{2}+q^{2}\right)}{\tilde{E}_{g}+\tilde{\delta}-\tilde{E}}, \quad \tilde{\lambda}=\frac{\tilde{\delta}}{\tilde{E}+\tilde{\delta}+\hbar^{2}\left(-\kappa^{2}+q^{2}\right) / 2 m_{h}},
$$

$\kappa$ равно модулю $x$-компоненты квазиимпульса $s o$-дырки под барьером, связь $\kappa$ с энергией дается выражением (4), примененным к широкозонному полупроводнику. $\tilde{D}_{1}$ и $\tilde{D}_{2}$ - нормировочные коэффициенты.

Для $x<a / 2$ волновые функции могут быть получены из (9) с учетом соотношения (7). Переход к функциям другой симметрии $(v=-1)$ осуществляется формальной заменой $\xi \leftrightarrow \eta$.

\section{4. Дискретный спектр so-дырок в квантовой яме}

Используя граничные условия для волновой функции $s o$-дырки, получаем дисперсионное уравнение:

$$
\left(\alpha \kappa \cot \frac{k a}{2}+k\right)\left(\alpha \kappa \tan \frac{k a}{2}-k\right)=q^{2}(\alpha \tilde{\lambda}+\lambda)^{2} .
$$

Здесь $\alpha=S_{d} / \tilde{S}_{d}$. Уравнение (10) имеет одно очевидное решение $E=-2 \delta$. В дальнейшем мы это решение опускаем, так как при $E=-2 \delta$ волновая функция $s o$-дырки тождественно равна нулю. Другие решения (10) следует искать численно. Важным свойством дисперсионного уравнения является то, что при заданном $q$ может существовать несколько значений $k$, удовлетворяющих (10), так что все его решения образуют несколько ветвей, каждая из которых характеризуется своей зависимостью $k(q)$ (рис. 2). Как и в задаче об одномерной симметричной квантовой яме, для (10) можно заведомо гарантировать существование хотя бы одной ветви спектра, даже если мощность ямы очень мала.

Как правило, все значения $k(q)$, лежащие на ветви спектра $s o$-дырок с номером $n$, содержатся в интервале от $(n-1) \pi / a$ до $n \pi / a$, поскольку на границах указанного интервала одна из тригонометрических функций,

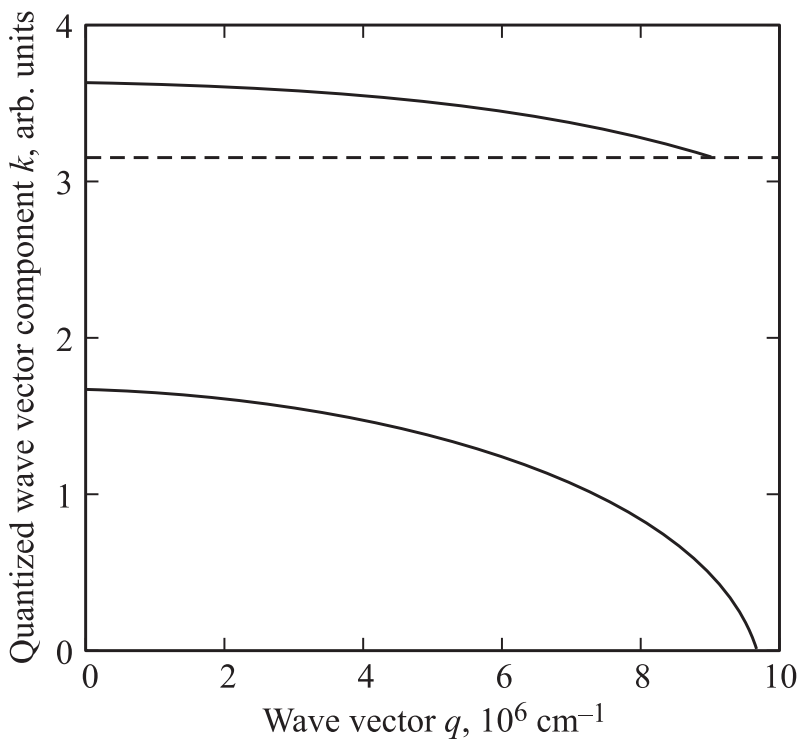

Рис. 2. Зависимости $k(q)$ для различных подзон размерного квантования в гетероструктуре $\mathrm{AlSb} / \mathrm{InAs}_{0.84} \mathrm{Sb}_{0.16} / \mathrm{AlSb}$ при ширине кватовой ямы $a=40 \AA$. Значения $k$ номированы на величину $1 / a$. Штриховая линия - прямая $k=\pi / a$.

входящих в (10), становится бесконечной. Фактически это означает, что при больших $q$ линии на плоскости $(q, k)$ должны быть почти прямыми.

Еще одним важным свойством (10) является возможность существования обрывов у ветвей спектра $s o$-дырок в яме. Как правило, обрывы линий $k(q)$ связаны с уменьшением $\kappa$ до нуля и делокализацией связанных состояний. В параболическом приближении (6) геометрическим местом точек на плоскости $(q, k)$, для которых выполнено неравенство $\kappa^{2}>0$, является эллипс:

$$
k^{2}=\frac{2 m_{s o} U_{s o}}{\hbar^{2}}-q^{2}\left(1-\frac{m_{s o}}{\tilde{m}_{s o}}\right) .
$$

Именно на пересечении границы эллипса с прямыми $k=n \pi / a$ обрываются ветви спектра на рис. 2. Предварительно эти ветви достаточно резко опускаются вниз, потому что только быстрым изменением $k$ должно компенсироваться убывание $\kappa$ в (10). В случае точного выражения (4) точки с $\kappa^{2}>0$ образуют область с более сложной формой.

Несмотря на то что состояния с различной четностью для $s o$-дырок в общем случае не разделяются, мы будем называть ветвь четной или нечетной, если таковым являлось ее состояние при $q=0$.

Положим

$$
d=\frac{q(\alpha \tilde{\lambda}+\lambda)}{\alpha \kappa \tan (k a / 2)-k}
$$

Как следует из граничных условий, $d$ является отношением $D_{2}$ к $D_{1}$. С помощью условия нормировки 
получаем $D_{1}$ :

$$
\begin{aligned}
D_{1}^{-2} & =\frac{a}{2}\left(1+d^{2}\right) N_{d 1}+\frac{\sin k a}{2 k}\left(1-d^{2}\right) N_{d 2} \\
& +2 q d \lambda(2-\lambda) \sin k a+\frac{\alpha^{2}}{\kappa}\left(d^{2} \sin ^{2} \frac{k a}{2}+\cos ^{2} \frac{k a}{2}\right) \tilde{N}_{d} \\
& -2 \alpha^{2} q d \lambda(2-\lambda) \sin k a .
\end{aligned}
$$

Здесь

$$
\begin{aligned}
& N_{d 1}=S_{d}^{2}+\left(k^{2}+q^{2}\right)\left(1+2 \lambda^{2}\right), \\
& N_{d 2}=S_{d}^{2}+\left(q^{2}-k^{2}\right)\left(1+2 \lambda^{2}\right), \\
& \tilde{N}_{d}=\tilde{S}_{d}^{2}+\left(-\kappa^{2}+q^{2}\right)\left(1+2 \tilde{\lambda}^{2}\right) .
\end{aligned}
$$

Из (10) следует, что для четных ветвей $s o$-дырок в яме, включая основную, при $q \rightarrow 0$ коэффициенты нормировки $D_{1}$ и $D_{2}$ ведут себя по-разному: $D_{1} \propto q$, тогда как $D_{2}$ в первом приближении остается приблизительно постоянным. Для нечетных ветвей при малых $q$ поведение коэффициентов нормировки, наоборот, следующее: $D_{2} \propto q, D_{1} \approx$ const.

\section{5. Волновые функции непрерывного спектра sо-дырок}

Построим волновые функции непрерывного спектра $s o$-дырок для $v=1$. Решения кейновской системы уравнений (2) при $-a / 2<x<a / 2$, соответствующие $v=1$, образуют двумерное линейное пространство так же, как это было и в случае дискретного спектра. Мы используем следующий базис для этого пространства:

$$
\begin{gathered}
\mathbf{V}_{1}=\left(\begin{array}{c}
i S_{c} \cos k x \eta \\
k \sin k x \eta-\lambda q \cos k x \xi \\
-i q \cos k x \eta+i \lambda k \sin k x \xi \\
-\lambda k \sin k x \xi+\lambda q \cos k x \eta
\end{array}\right), \\
\mathbf{V}_{2}=\left(\begin{array}{c}
i S_{c} \sin k x \xi \\
-k \cos k x \xi-\lambda q \sin k x \eta \\
-i \lambda k \cos k x \eta-i q \sin k x \xi \\
-\lambda q \sin k x \xi-\lambda k \cos k x \eta
\end{array}\right),
\end{gathered}
$$

где

$$
S_{c}=\hbar \gamma\left(k^{2}+q^{2}\right) /(E g+\delta-E)
$$

- амплитуда колебаний $s$-компоненты волновой функции в области ямы.

Над барьером, при $x>a / 2$, мы имеем четыре базисных вектора $\tilde{x}=x-a / 2$ :

$$
\tilde{\mathbf{V}}_{1}=\left(\begin{array}{c}
i \tilde{S}_{c} \cos \tilde{k} \tilde{x} \eta \\
\tilde{k} \sin \tilde{k} \tilde{x} \eta-\lambda q \cos \tilde{k} \tilde{x} \xi \\
-i q \cos \tilde{k} \tilde{x} \eta+i \lambda \tilde{k} \sin \tilde{k} \tilde{x} \xi \\
-\lambda \tilde{k} \sin \tilde{k} \tilde{x} \xi+\lambda q \cos \tilde{k} \tilde{x} \eta
\end{array}\right),
$$

$$
\begin{aligned}
& \tilde{\mathbf{V}}_{2}=\left(\begin{array}{c}
i \tilde{S}_{c} \sin \tilde{k} \tilde{x} \xi \\
-\tilde{k} \cos \tilde{k} \tilde{x} \xi-\lambda q \sin \tilde{k} \tilde{x} \eta \\
-i \lambda \tilde{k} \cos \tilde{k} \tilde{x} \eta-i q \sin \tilde{k} \tilde{x} \xi \\
-\lambda q \sin \tilde{k} \tilde{x} \xi-\lambda \tilde{k} \cos \tilde{k} \tilde{x} \eta
\end{array}\right), \\
& \tilde{\mathbf{V}}_{3}=\left(\begin{array}{c}
i \tilde{S}_{c} \cos \tilde{k} \tilde{x} \xi \\
\tilde{k} \sin \tilde{k} \tilde{x} \xi-\lambda q \cos \tilde{k} \tilde{x} \eta \\
-i q \cos \tilde{k} \tilde{x} \xi+i \lambda \tilde{k} \sin \tilde{k} \tilde{x} \eta \\
-\lambda \tilde{k} \sin \tilde{k} \tilde{x} \eta+\lambda q \cos \tilde{k} \tilde{x} \xi
\end{array}\right), \\
& \tilde{\mathbf{V}}_{4}=\left(\begin{array}{c}
i \tilde{S}_{c} \sin \tilde{k} \tilde{x} \eta \\
-\tilde{k} \cos \tilde{k} \tilde{x} \eta-\lambda q \sin \tilde{k} \tilde{x} \xi \\
-i \lambda \tilde{k} \cos \tilde{k} \tilde{x} \xi-i q \sin \tilde{k} \tilde{x} \eta \\
-\lambda q \sin \tilde{k} \tilde{x} \eta-\lambda \tilde{k} \cos \tilde{k} \tilde{x} \xi
\end{array}\right) .
\end{aligned}
$$

Здесь и далее $\tilde{k}-$ вектор so-дырки, а $\tilde{S}_{c}=$ $=\hbar \gamma\left(\tilde{k}^{2}+q^{2}\right) /\left(\tilde{E}_{g}+\tilde{\delta}-\tilde{E}\right)-$ амплитуда $s$-компоненты волновой функции в области барьера. Для построения базиса пространства волновых функций so-дырок на всей оси $x$ можно поступить следующим образом: выбрать один вектор из $\mathbf{V}_{1}, \mathbf{V}_{2}$ и „сшить“, исходя из граничных условий, с суперпозицией, составленной из векторов $\tilde{\mathbf{V}}_{1}, \tilde{\mathbf{V}}_{2}, \tilde{\mathbf{V}}_{3}, \tilde{\mathbf{V}}_{4}$. Найдем $\Psi_{1}-$ первую базисную функцию этого пространства. В области квантовой ямы при $-a / 2<x<a / 2$ положим $\Psi_{1}=\mathbf{V}_{1}$, а в области барьера $(x>a / 2) \Psi_{1}=c_{11} \tilde{\mathbf{V}}_{1}+c_{12} \tilde{\mathbf{V}}_{2}+c_{13} \tilde{\mathbf{V}}_{3}+c_{14} \tilde{\mathbf{V}}_{4}$. Граничные условия представляют собой систему уравнений для нахождения коэффициентов $c_{11}, c_{12}, c_{13}, c_{14}$. В итоге получаем

$$
\begin{gathered}
c_{11}=-\alpha_{c} \cos \frac{k a}{2}, \quad c_{12}=\frac{q}{\tilde{k}}\left(\lambda+\alpha_{c} \tilde{\lambda}\right) \cos \frac{k a}{2}, \\
c_{13}=0, \quad c_{14}=-\frac{k}{\tilde{k}} \sin \frac{k a}{2} .
\end{gathered}
$$

Для $s o$-дырок непрерывного спектра $\alpha_{c}=-S_{c} / \tilde{S}_{c}$.

Для нахождения второй базисной функции $\Psi_{2}$ мы поступаем аналогичным образом. Выражения для $c_{21}, c_{22}, c_{23}, c_{24}$ имеют вид

$$
\begin{aligned}
c_{21}=0, \quad c_{22} & =\frac{k}{\tilde{k}} \sin \frac{k a}{2}, \quad c_{23}=-\alpha_{c} \sin \frac{k a}{2}, \\
c_{24} & =\frac{q}{\tilde{k}}\left(\lambda+\alpha_{c} \tilde{\lambda}\right) \sin \frac{k a}{2} .
\end{aligned}
$$

Волновые функции $\Psi_{1}$ и $\Psi_{2}$ не ортогональны. Их скалярное произведение есть

$$
\Pi=\int_{-L}^{L} \Psi_{1} \Psi_{2}^{*} d x=2 L \frac{k q \tilde{N}_{c}}{2 \tilde{k}^{2}}\left(\lambda+\alpha_{c} \tilde{\lambda}\right) \cos k a,
$$

где $\tilde{N}_{c}=\tilde{S}_{c}^{2}+\left(\tilde{k}^{2}+q^{2}\right)\left(1+2 \tilde{\lambda}^{2}\right)$. В (14) и далее мы используем нормировку в ящике с длиной стороны $2 L$. Чтобы нормировать огибающие волновых функций $\Psi_{1}$ 
и $\Psi_{2}$, их следует домножить на коэффициенты $C_{1}$ и $C_{2}$, определяемые следующими формулами:

$$
\begin{aligned}
& C_{1}=\frac{\tilde{k}}{\sqrt{2 L}} \sqrt{\frac{2}{\tilde{N}_{c}}} \\
& \times\left[\alpha_{c}^{2} \tilde{k}^{2} \cos ^{2} \frac{k a}{2}+q^{2}\left(\lambda+\alpha_{c} \tilde{\lambda}\right)^{2} \cos ^{2} \frac{k a}{2}+k^{2} \sin ^{2} \frac{k a}{2}\right]^{-1 / 2}, \\
& C_{2}=\frac{\tilde{k}}{\sqrt{2 L}} \sqrt{\frac{2}{\tilde{N}_{c}}} \\
& \times\left[\alpha_{c}^{2} \tilde{k}^{2} \sin ^{2} \frac{k a}{2}+q^{2}\left(\lambda+\alpha_{c} \tilde{\lambda}\right)^{2} \sin ^{2} \frac{k a}{2}+k^{2} \cos ^{2} \frac{k a}{2}\right]^{-1 / 2} .
\end{aligned}
$$

Ортонормированный базис волновых функций so-дырок непрерывного спектра состоит из волновых функций $\Psi_{c 1}$ и $\Psi_{c 2}$, соответствующих двум стоячим волнам:

$$
\begin{gathered}
\Psi_{c 1}=C_{1} \Psi_{1} \\
\Psi_{c 2}=\frac{1}{\sqrt{1-\Pi^{2} C_{1}^{2} C_{2}^{2}}} C_{2} \Psi_{2}-\frac{\Pi C_{1} C_{2}}{\sqrt{1-\Pi^{2} C_{1}^{2} C_{2}^{2}}} C_{1} \Psi_{1} .
\end{gathered}
$$

Еще раз упомянем, что кроме волновых функций с $v=1$ существуют еще и волновые функции с $v=-1$. Таким образом, кратность вырождения уровней энергии so-дырок в непрерывном спектре равна четырем.

\section{4. Матричные элементы переходов}

Предположим, что плоская монохроматическая электромагнитная волна линейно поляризована, а ее векторный потенциал равен

$$
\mathbf{A}=\mathbf{A}_{0} e^{i(\mathbf{k} / \mathbf{r}-\omega t)},
$$

$\mathbf{k}_{l}$ - волновой вектор волны, $k_{l} \ll 1 / a$ и пространственной зависимостью векторного потенциала на расстояниях порядка ширины квантовой ямы можно пренебречь. Матричный элемент исследуемого оптического перехода равен

$$
\begin{aligned}
M & =\frac{\hbar e}{m_{0} c} \int \psi_{h}^{*}\left(\mathbf{A}_{0} \nabla\right) \psi d^{3} \mathbf{r} \\
& =\frac{\gamma e}{c} \delta\left(\mathbf{q}_{h}-\mathbf{q}\right) \int\left(\mathbf{A}_{0} \Psi_{h, p}^{*}\right) \Psi_{s} d x,
\end{aligned}
$$

где мы учли, что интегрирование фазовых множителей волновых функций по $y$ и $z$ дает $\delta$-функцию [19], а также то, что отличными от нуля являются следующие матричные элементы:

$$
\left\langle x\left|\frac{\partial}{\partial x}\right| s\right\rangle / V=\left\langle x\left|\frac{\partial}{\partial y}\right| y\right\rangle / V=\left\langle x\left|\frac{\partial}{\partial z}\right| z\right\rangle / V=\frac{m_{0} \gamma}{\hbar} .
$$

Так как волновые функции тяжелых дырок быстро затухают под барьером вследствие их большой эффективной массы, будем считать, что поглощение излучения происходит только внутри квантовой ямы, и поэтому в (17) ограничимся интегрированием в пределах от $-a / 2$ до $a / 2$. Выразим матричный элемент через интегралы перекрытия между $s$-компонентой волновой функции so-дырки и $i$-компонентой $(i=x, y, z)$ волновой функции тяжелой дырки:

$$
M^{j}=M_{x}^{j}+M_{y}^{j}+M_{z}^{j}=\frac{\gamma e A_{x}}{c} I_{x}^{j}+\frac{\gamma e A_{y}}{c} I_{y}^{j} \frac{\gamma e A_{z}}{c} I_{z}^{j},
$$

где

$$
I_{i}=\int_{-a / 2}^{a / 2} \Psi_{h, i}^{*} \Psi_{s} d x
$$

Верхний индекс $j=d, c 1, c 2$ в (18) принимает значение $d$, если конечное состояние лежит в дискретном спектре $s o$-дырок, и значение $c 1$ или $c 2$, если конечное состояние описывается волновой функцией $\Psi_{c 1}$ или $\Psi_{c 2}$ соответственно. В тех случаях, когда необходимо указать, меняется ли симметрия дырки при переходе, мы будем добавлять к величинам в (18) верхний индекс $s$, если симметрия не меняется (т.е. $v_{h}=v$, где квантовое число $v_{h}$ обозначает, по аналогии с $v$, симметрию волновой функции тяжелых дырок), и индекс $a$, если симметрия меняется $\left(v_{h}=-v\right)$.

Как будет показано в разд. 5.1, интерференционные члены, возникающие при возведении в квадрат выражения (18), не дают вклада в общее число переходов.

Выпишем ненулевые интегралы перекрытия для переходов с четных уровней тяжелых дырок в дискретный спектр so-дырок:

$$
\begin{gathered}
I_{x}^{d, a}=i H_{1} D_{1} q S_{d}\left(\frac{\sin \left[\left(k_{h}-k\right) a / 2\right]}{k_{h}-k}+\frac{\sin \left[\left(k_{h}+k\right) a / 2\right]}{k_{h}+k}\right), \\
I_{y}^{d, s}=-H_{1} D_{2} k_{h} S_{d}\left(\frac{\sin \left[\left(k_{h}-k\right) a / 2\right]}{k_{h}-k}-\frac{\sin \left[\left(k_{h}+k\right) a / 2\right]}{k_{h}+k}\right), \\
I_{z}^{d, s}=I_{x}^{d, a}+i I_{y}^{d, s} .
\end{gathered}
$$

Интегралы перекрытия для переходов с нечетных уровней тяжелых дырок в дискретный спектр so-дырок есть

$$
\begin{gathered}
I_{x}^{d, a}=i H_{2} D_{2} q S_{d}\left(\frac{\sin \left[\left(k_{h}-k\right) a / 2\right]}{k_{h}-k}-\frac{\sin \left[\left(k_{h}+k\right) a / 2\right]}{k_{h}+k}\right), \\
I_{y}^{d, s}=H_{2} D_{1} k_{h} S_{d}\left(\frac{\sin \left[\left(k_{h}-k\right) a / 2\right]}{k_{h}-k}+\frac{\sin \left[\left(k_{h}+k\right) a / 2\right]}{k_{h}+k}\right), \\
I_{z}^{d, s}=-I_{x}^{d, a}-i I_{y}^{d, s} .
\end{gathered}
$$

Интегралы перекрытия для переходов с четных уровней тяжелых дырок в непрерывный спектр so-дырок есть

$$
\begin{gathered}
I_{x}^{c 1, a}=i H_{1} C_{1} q S_{c}\left(\frac{\sin \left[\left(k_{h}-k\right) a / 2\right]}{k_{h}-k}+\frac{\sin \left[\left(k_{h}+k\right) a / 2\right]}{k_{h}+k}\right), \\
I_{x}^{c 2, a}=-\frac{\Pi C_{1} C_{2}}{\sqrt{1-\Pi^{2} C_{1}^{2} C_{2}^{2}}} I_{x}^{c 1, a},
\end{gathered}
$$




$$
\begin{aligned}
& I_{y}^{c 2, s}=- \frac{C_{2} H_{1} S_{c} k_{h}}{\sqrt{1-\Pi^{2} C_{1}^{2} C_{2}^{2}}} \\
& \times\left(\frac{\sin \left[\left(k_{h}-k\right) a / 2\right]}{k_{h}-k}-\frac{\sin \left[\left(k_{h}+k\right) a / 2\right]}{k_{h}+k}\right), \\
& I_{z}^{c 1, s}=I_{x}^{c 1, a}, \\
& I_{z}^{c 2, s}=I_{x}^{c 2, a}+i I_{y}^{c 2, s} .
\end{aligned}
$$

Интегралы перекрытия для переходов с нечетных уровней тяжелых дырок в непрерывный спектр so-дырок есть

$$
\begin{gathered}
I_{x}^{c 2, a}=\frac{i S_{c} C_{2} H_{2} q}{\sqrt{1-\Pi^{2} C_{1}^{2} C_{2}^{2}}} \\
\times\left(\frac{\sin \left[\left(k_{h}-k\right) a / 2\right]}{k_{h}-k}-\frac{\sin \left[\left(k_{h}+k\right) a / 2\right]}{k_{h}+k}\right), \\
I_{y}^{c 1, s}=C_{1} H_{2} S_{c} k_{h}\left(\frac{\sin \left[\left(k_{h}-k\right) a / 2\right]}{k_{h}-k}+\frac{\sin \left[\left(k_{h}+k\right) a / 2\right]}{k_{h}+k}\right), \\
I_{y}^{c 2, s}=\frac{\Pi C_{1} C_{2}}{\sqrt{1-\Pi^{2} C_{1}^{2} C_{2}^{2}}} I_{y}^{c 1, s}, \\
I_{z}^{c 1, s}=-i I_{y}^{c 1, s}, \\
I_{z}^{c 2, s}=-I_{x}^{c 2, a}-i I_{y}^{c 2, s} .
\end{gathered}
$$

\section{5. Число переходов}

\section{1. Полное число переходов и его зависимость от угла поляризации}

Полное число переходов, стимулированных плоской монохроматической волной, на единицу площади в единицу времени равно

$$
Q=\frac{2 \pi}{\hbar} \sum_{i, f}\left|M_{i f}\right|^{2} f\left(E_{i}\right) \delta\left(E_{i}-E_{f}-\hbar \omega\right),
$$

где $f\left(E_{i}\right)$ - фермиевская функция распределения дырок по энергиям. Суммирование в (23) ведется по начальным $(i)$ и конечным $(f)$ состояниям. Начальные состояния лежат в дискретном спектре тяжелых дырок, a конечные состояния - в подзоне $s o$-дырок. При расчете числа переходов мы считали состояния в спинотщепленной зоне полностью свободными.

Фермиевская функция распределения параметрически зависит от квазиуровня Ферми валентной зоны $\mu$, который связан с двумерной концентрацией дырок $p$ уравнением

$$
p=\frac{m_{h} T}{\pi \hbar^{2}} \sum_{k_{h}} \ln \left[1+\exp \left(\frac{\delta}{T}-\frac{\hbar^{2} k_{h}^{2}}{2 m_{h} T}-\frac{\mu}{T}\right)\right] .
$$

Полное число переходов $Q$ можно представить в виде суммы двух слагаемых, $Q^{d}$ и $Q^{c}$, первое из которых соответствует количеству переходов в дискретный спектр so-дырок, а второе - количеству переходов в непрерывный спектр so-дырок:

$$
\begin{aligned}
Q^{d} & =\frac{2 \pi}{\hbar} \sum_{\nu_{h}, k_{h}} \int_{0}^{\infty} \frac{q d q}{(2 \pi)^{2}} \int_{0}^{2 \pi} d \varphi \sum_{\nu, k(q)}\left|M^{d}\right|^{2} f\left(E_{h}\right) \delta\left(E_{h}-E-\hbar \omega\right), \\
Q^{c} & =\frac{2 \pi}{\hbar} \sum_{\nu_{h}, k_{h}} \int_{0}^{\infty} \frac{q d q}{(2 \pi)^{2}} \int_{0}^{2 \pi} d \varphi \\
& \times \sum_{\nu} \int_{k_{\min }}^{\infty} \frac{d k}{2 \pi} 2 L\left(\left|M^{c 1}\right|^{2}+\left|M^{c 2}\right|^{2}\right) f\left(E_{h}\right) \delta\left(E_{h}-E-\hbar \omega\right) .
\end{aligned}
$$

Здесь $\varphi-$ угол между $\mathbf{A}_{\|}$(проекцией $\mathbf{A}_{0}$ на плоскость $y z$ ) и осью $y$. В (25) и (26) суммирование ведется по ветвям дискретного спектра тяжелых дырок, а также по числам $v_{h}= \pm 1$ и $v= \pm 1$, характеризующим возможные симметрии волновых функций; кроме того, в (25) проводится суммирование по ветвям дискретного спектра so-дырок, а в (26) по всем значениям $k$, соответствующим состояниям в непрерывном спектре $s o$-дырок. Величина $k_{\min }$ зависит от $q$ и определяется из условия $\kappa=0$.

В (25) и (26) от угла $\varphi$ зависят только матричные элементы. Выберем какой-либо один из них. Квадрат его модуля есть

$$
\left|M^{j}\right|^{2}=\left|M_{x}^{j}\right|^{2}+\left|M_{y}^{j}\right|^{2}+\left|M_{z}^{j}\right|^{2}+M_{x}^{j}\left(M_{z}^{j}\right)^{*}+M_{z}^{j}\left(M_{x}^{j}\right)^{*} .
$$

При получении (27) был использован тот факт, что фаза $M_{y}^{j}$ отличается как от фазы $M_{x}^{j}$, так и от фазы $M_{z}^{j}$ на $\pi / 2$. Проинтегрируем равенство (27) по углу $\varphi$. Из того, что $M_{x}^{j}$ не зависит от $\varphi$, а $M_{z}^{j} \propto \sin \varphi$, следует

$$
\int_{0}^{2 \pi}\left[M_{x}^{j}\left(M_{z}^{j}\right)^{*}+M_{z}^{j}\left(M_{x}^{j}\right)^{*}\right] d \varphi=0 .
$$

Учитывая пропорциональность $M_{y}^{j} \propto \cos \varphi$, получаем

$$
\int_{0}^{2 \pi}\left|M^{j}\right|^{2}=2 \pi\left|M_{x}^{j}\right|^{2}+\pi\left|M_{y}^{j}(\varphi=0)\right|^{2}+\pi\left|M_{z}^{j}(\varphi=0)\right|^{2} .
$$

Чтобы отыскать зависимость числа переходов $Q$ от поляризации падающего излучения, перепишем (28), используя интегралы перекрытия $I_{i}^{j}$ :

$$
\begin{aligned}
& \int_{0}^{2 \pi}\left|M^{j}\right|^{2}=\frac{\gamma^{2} e^{2} A_{0}^{2 c}}{c^{2}} \\
& \quad \times\left[2 \pi\left|I_{x}^{j, a}\right|^{2} \cos ^{2} \beta+\pi\left(\left|I_{y}^{j, s}\right|^{2}+\left|I_{z}^{j, s}\right|^{2}\right) \sin ^{2} \beta\right],
\end{aligned}
$$


где $\beta$ - угол между $\mathbf{A}_{0}$ и осью $x$. Просуммируем (29) по $v_{h}$ и $v$ :

$$
\begin{aligned}
& \sum_{\nu_{h}, v} \int_{0}^{2 \pi}\left|M^{j}\right|^{2}=\frac{2 \gamma^{2} e^{2} A_{0}^{2 c}}{c^{2}} \\
& \quad \times\left[2 \pi\left|I_{x}^{j, a}\right|^{2} \cos ^{2} \beta+\pi\left(\left|I_{y}^{j, s}\right|^{2}+\left|I_{z}^{j, s}\right|^{2}\right) \sin ^{2} \beta\right] .
\end{aligned}
$$

Введем следующие обозначения:

$$
\begin{gathered}
\sigma_{\perp}^{j}=2 \pi\left|I_{x}^{j, a}\right|^{2}, \\
\sigma_{\|}^{j}=\pi\left(\left|I_{y}^{j, s}\right|^{2}+\left|I_{z}^{j, s}\right|^{2}\right) .
\end{gathered}
$$

Используемые в (31) индексы $\perp$ и $\|$ соответствуют двум частным случаям поляризации: $\beta=0$ и $\beta=\pi / 2$. Для максимального упрощения (26) положим

$$
\sigma_{p}^{c}=2 L\left(\sigma_{p}^{c 1}+\sigma_{p}^{c 2}\right) .
$$

В (32) и далее индекс $p$ принимает значения $\perp$ и $\|$. Подставив (31) и (32) в (25) и (26), получим:

$$
\begin{aligned}
Q_{p}^{d}= & \frac{4 \pi \gamma^{2} e^{2} A_{0}^{2}}{\hbar c^{2}} \\
& \times \sum_{k_{h}} \int_{0}^{\infty} \frac{q d q}{(2 \pi)^{2}} \sum_{k(q)} \sigma_{p}^{d} f\left(E_{h}\right) \delta\left(E_{h}-E-\hbar \omega\right), \\
Q_{p}^{c}= & \frac{4 \pi \gamma^{2} e^{2} A_{0}^{2}}{\hbar c^{2}} \\
\times & \sum_{k_{h}} \int_{0}^{\infty} \frac{q d q}{(2 \pi)^{2}} \int_{k_{\min }}^{\infty} \sigma_{p}^{c} f\left(E_{h}\right) \delta\left(E_{h}-E-\hbar \omega\right) \frac{d k}{2 \pi} .
\end{aligned}
$$

Зная $Q_{p}^{d}$ и $Q_{p}^{c}$, можно найти полное число переходов $Q$ при произвольном $\beta$ :

$$
Q(\beta)=\left(Q_{\perp}^{d}+Q_{\perp}^{c}\right) \cos ^{2} \beta+\left(Q_{\|}^{d}+Q_{\|}^{c}\right) \sin ^{2} \beta .
$$

\section{2. Число переходов тяжелых дырок в дискретный спектр so-дырок}

Чтобы воспользоваться свойствами $\delta$-функции и упростить (33), следует заменить в (33) интегрирование по $q$ на интегрирование по $E_{h}-E$. Якобиан такой замены переменной равен

$$
\frac{d E_{h}(q)}{d q}-\frac{d E_{h}(q)}{d q}=2 q\left[\frac{d E_{h}\left(q^{2}\right)}{d q^{2}}-\frac{d E_{h}\left(q^{2}\right)}{d q^{2}}\right],
$$

a (33) принимает вид

$$
Q_{p}^{d}=\frac{\gamma^{2} e^{2} A_{0}^{2}}{2 \pi \hbar c^{2}} \sum_{k_{h}} \sum_{E_{h}} \frac{\sigma_{p}^{d} f\left(E_{h}\right)}{\left|E_{h}^{\prime}\left(q^{2}\right)-E^{\prime}\left(q^{2}\right)\right|} .
$$

Внутреннее суммирование в (37) ведется по тем энергиям $E_{h}$, при которых аргумент $\delta$-функции из (33) обращается в нуль. Поиск таких $E_{h}$ может быть осуществлен только численными методами. Представим схему проверки некоторого пробного значения $E_{h}$ на предмет выполнения равенства $E_{h}-E-\hbar \omega=0$. Сначала мы вычисляем $q$ с помощью (3), после чего получаем $k$ и $\kappa$ из формулы (4), примененной к области квантовой ямы и к области барьера. Завершающим шагом проверки является подстановка рассчитанных величин в (11) и сравнение левой и правой частей дисперсионного уравнения. Заметим, что в (37) суммирование по $E_{h}$ почти полностью соответствует суммированию по ветвям дискретного спектра $s o$-дырок в яме. Некоторая разница возникает из-за того, что при заданных $\omega$ и $k_{h}$ может иметься и более одного канала для перехода на выбранную ветвь $s o$-дырок.

Зависимость энергии тяжелых дырок от волнового вектора в яме квадратична (3), поэтому $d E_{h}\left(q^{2}\right) / d q^{2}=$ $=-\hbar^{2} / 2 m_{h}$.

При малых $q$ энергию $s o$-дырок в яме можно разложить в ряд с точностью до квадратичного члена. Ввиду того что дисперсионное уравнение (11) четно по $q$, волновой вектор $k(q) \approx k(0)+k^{\prime \prime}(0)\left(q^{2} / 2\right)$, а энергия so-дырок в яме равна

$$
E\left(q^{2}\right) \approx-2 \delta-\frac{\hbar^{2} k^{2}(0)}{2 m_{s o}}-\frac{\hbar^{2}\left[1+k(0) k^{\prime \prime}(0)\right] q^{2}}{2 m_{s o}} .
$$

Для зависимости (38) роль эффективной массы играет величина $m_{s o}^{\text {eff }}$, которая отличается от $m_{s o}$ :

$$
m_{s o}^{\mathrm{eff}}=\frac{m_{s o}}{1+k(0) k^{\prime \prime}(0)}
$$

В приближении (38) число переходов определяется с помощью следующей формулы:

$$
Q_{p}^{d}=\frac{\gamma^{2} e^{2} A_{0}^{2}}{\pi \hbar^{3} c^{2}} \sum_{k_{h}} \sum_{k(0)} m_{r}^{d} \sigma_{p}^{d} f\left(E_{h}\right),
$$

где $m_{r}=m_{h} m_{s o}^{\text {eff }} /\left|m_{h}-m_{s o}^{\text {eff }}\right|-$ величина, аналогичная приведенной массе. Внутреннее суммирование в (40) ведется уже по ветвям so-дырок в яме. Переходы в (40) происходят при $q=\sqrt{m_{r}^{d}\left(\omega-\omega_{\mathrm{th}}^{d}\right) / \hbar}$, где $\omega_{\mathrm{th}}^{d}\left(k_{h}, k\right)-$ пороговая частота поглощения:

$$
\hbar \omega_{\mathrm{th}}^{d}\left(k_{h}, k\right)=\Delta-\frac{\hbar^{2} k_{h}^{2}}{2 m_{h}}+\frac{\hbar^{2} k^{2}(0)}{2 m_{s o}} .
$$

Особое значение для поглощения имеют основные уровни энергии тяжелых и $s o$-дырок. При малых значениях ширины квантовой ямы пороговая энергия поглощения, связанного с основными уровнями, близка к $\tilde{\Delta}$, а при больших значениях стремится к $\Delta$.

Еще одно отличие внутризонного поглощения от межзонного заключается в следующем. Как видно из (41), пороговая энергия поглощения, связанного с основными уровнями, не является минимальной из всего набора пороговых энергий, потому что для ветвей спектра тяжелых дырок с большими номерами $\hbar \omega_{\text {th }}^{d}\left(k_{h}, k\right)$ меньше. Несмотря на то что поглощение, связанное с этими уровнями, не такое сильное из-за их сравнительно малой заселенности, при больших $a$ оно должно существенно сглаживать край поглощения, связанного с основными уровнями спектра тяжелых и so-дырок. 


\section{3. Число переходов тяжелых дырок В непрерывный спектр sо-дырок}

Заменим в (34) переменные интегрирования $q, k$ на переменные $E_{h}, E_{h}-E$. В связи с тем что $\partial E_{h} / \partial k=0$, якобиан такой замены оказывается равным

$$
\frac{\partial E_{h}}{\partial q} \frac{\partial\left(E_{h}-E\right)}{\partial k}=-\frac{\hbar^{2} q}{2 m_{h}}\left(2 k \frac{\partial E}{\partial K^{2}}\right) .
$$

Подставляя (42) в (34) и интегрируя с помощью $\delta$-функции, получаем

$$
Q_{p}^{c}=\frac{\gamma^{2} e^{2} m_{h} A_{0}^{2}}{4 \pi^{2} \hbar^{3} c^{2}} \sum_{k_{h}} \int_{E_{h}^{\min }}^{E_{h}^{\max }} \frac{\sigma_{p}^{c} f\left(E_{h}\right)}{k\left|E^{\prime}\left(K^{2}\right)\right|} d E_{h} .
$$

В (43) интегрирование проводится по отрезку $\left[E_{h}^{\min }, E_{h}^{\max }\right]$ - области определения подынтегрального выражения. Для $E_{h}^{\max }$ верно

$$
E_{h}^{\max }\left(k_{h}\right)=\delta-\frac{\hbar^{2} k_{h}^{2}}{2 m_{h}} .
$$

При $E_{h} \leq E_{h}^{\max }$ латеральная компонента квазиимпульса равна

$$
q=\sqrt{\frac{2 m_{h}\left(E_{h}^{\max }-E_{h}\right)}{\hbar^{2}}}
$$

Чтобы поглотившие фотоны носители заряда оказывались именно в непрерывном спектре $s o$-дырок, частота фотонов должна удовлетворять неравенству $\omega>\omega_{\mathrm{th}}^{c}\left(k_{h}\right)$. Пороговая частота $\omega_{\mathrm{th}}^{c}\left(k_{h}\right)$ процесса равна

$$
\omega_{\mathrm{th}}^{c}\left(k_{h}\right)=\Delta+U_{s o}-\frac{\hbar^{2} k_{h}^{2}}{2 m_{b}} .
$$

С ростом ширины квантовой ямы происходит увеличение $\omega_{\mathrm{th}}^{c}\left(k_{h}\right)$ из-за уменьшения $k_{h}$. Отметим, что пороговая частота межзонного поглощения с ростом ширины квантовой ямы ведет себя противоположным образом.

На левом конце $E_{h}^{\min }$ области определения подынтегрального выражения $\tilde{k}=0$.

Для $E_{h}^{\min }$ имеем уравнение

$$
\tilde{K}^{2}\left(E^{\min }\right)=\frac{2 m_{h}}{\hbar^{2}}\left(E_{h}^{\max }-E_{h}^{\min }\right),
$$

где $E^{\mathrm{min}}=E_{h}^{\min }-\hbar \omega$. Можно показать, что при $\omega>\omega_{\mathrm{th}}^{c}\left(k_{h}\right)$ решение уравнения (46) удовлетворяет неравенству $E_{h}^{\min } \leq E_{h}^{\max }$. В параболическом приближении (6) уравнение (46) можно разрешить:

$$
E_{h}^{\min }(\omega)=E_{h}^{\max }=\frac{\tilde{m}_{r}}{m_{h}}\left[\hbar \omega-\hbar \omega_{\text {th }}^{c}\left(k_{h}\right)\right]
$$

Здесь $\tilde{m}_{r}=m_{h} \tilde{m}_{s o} /\left(m_{h}-m_{s o}\right)-$ величина, аналогичная приведенной массе. Мы видим, что с увеличением частоты фотона размер области интегрирования в (43) растет. Однако при больших частотах этот рост не имеет никакого значения, поскольку с уменьшением $E_{h}$ заселенность энергетических уровней тяжелых дырок $f\left(E_{h}\right)$ экспоненциально убывает.

\section{6. Коэффициент поглощения}

Число поглощенных в единицу времени фотонов на единице площади квантовой ямы равно числу переходов $Q$. Внутризонный коэффициент поглощения равен

$$
G=\frac{Q}{N v}=\frac{2 \pi \hbar c}{n \omega} \frac{Q}{A_{0}^{2}} .
$$

Этот коэффициент безразмерен. Здесь $N-$ концентрация фотонов, $v=c / n, c-$ скорость света, $n-$ показатель преломления. В (48) использована связь между концентрацией $N$ и амплитудой векторного потенциала $A_{0}[19]$.

В литературе большее распространение получил внутризонный коэффициент поглощения, измеряемый в $\mathrm{CM}^{-1}$,

$$
\alpha=\frac{G}{a}=\frac{2 \pi \hbar c}{n \omega a} \frac{Q}{A_{0}^{2}} .
$$

Внутризонный коэффициент поглощения в форме (49), домноженный на фактор оптического ограничения Г, оказывается равным показателю экспоненты, которой описывается закон ослабления интенсивности излучения, проходящего через полупроводниковую гетероструктуру. В дальнейшем под коэффициентом поглощения мы будем подразумевать именно $\alpha$.

\section{1. Коэффициент поглощения, обусловленный переходом в дискретный спектр so-дырок}

Коэффициент поглощения при переходах в дискретный спектр, $\alpha_{p}^{d}$, согласно (49) и (39), равен

$$
\alpha_{p}^{d}=\frac{2 \gamma^{2} e^{2}}{\hbar^{2} c} \frac{1}{n \omega a} \sum_{k_{h}} \sum_{k(0)} m_{r}^{d} \sigma_{p}^{d} f\left(E_{h}\right) .
$$

Проведем анализ поведения коэффициента поглощения $\alpha_{p}^{d}$ в зависимости от параметров задачи. Для удобства проведения анализа мы будем считать, что выполнены неравенства $q_{T} \ll k_{h}, q_{T} \ll k$, где $q_{T}-$ волновой вектор теплового движения тяжелых дырок. Эти неравенства выполняются при достаточно больших значениях $U_{v}$ и $U_{s o}$ и не слишком больших значениях температуры, ширины квантовой ямы и концентрации дырок. В этом случае можно считать, что заселен только основной уровень тяжелых дырок и суммирование по $k_{h}$ в (50) можно опустить.

Для переходов с энергией, близкой к $\hbar \omega_{\mathrm{th}}^{d}\left(k_{h}, k\right)$, заселенность $f\left(E_{h}\right)$ можно считать постоянной, а $\alpha_{p}^{d}$, в соответствии с (23), повторяет поведение квадрата матричного элемента. При этом $q^{2}$ оказывается пропорциональным $\omega-\omega_{\mathrm{th}}^{d}\left(k_{h}, k\right)$, и это позволяет нам представить интегралы перекрытия (19), (20) в виде их первых членов при разложении в ряд Тейлора по степеням $q$. В случае статистики Больцмана для переходов, происходящих с основной ветви тяжелых дырок на четные ветви 

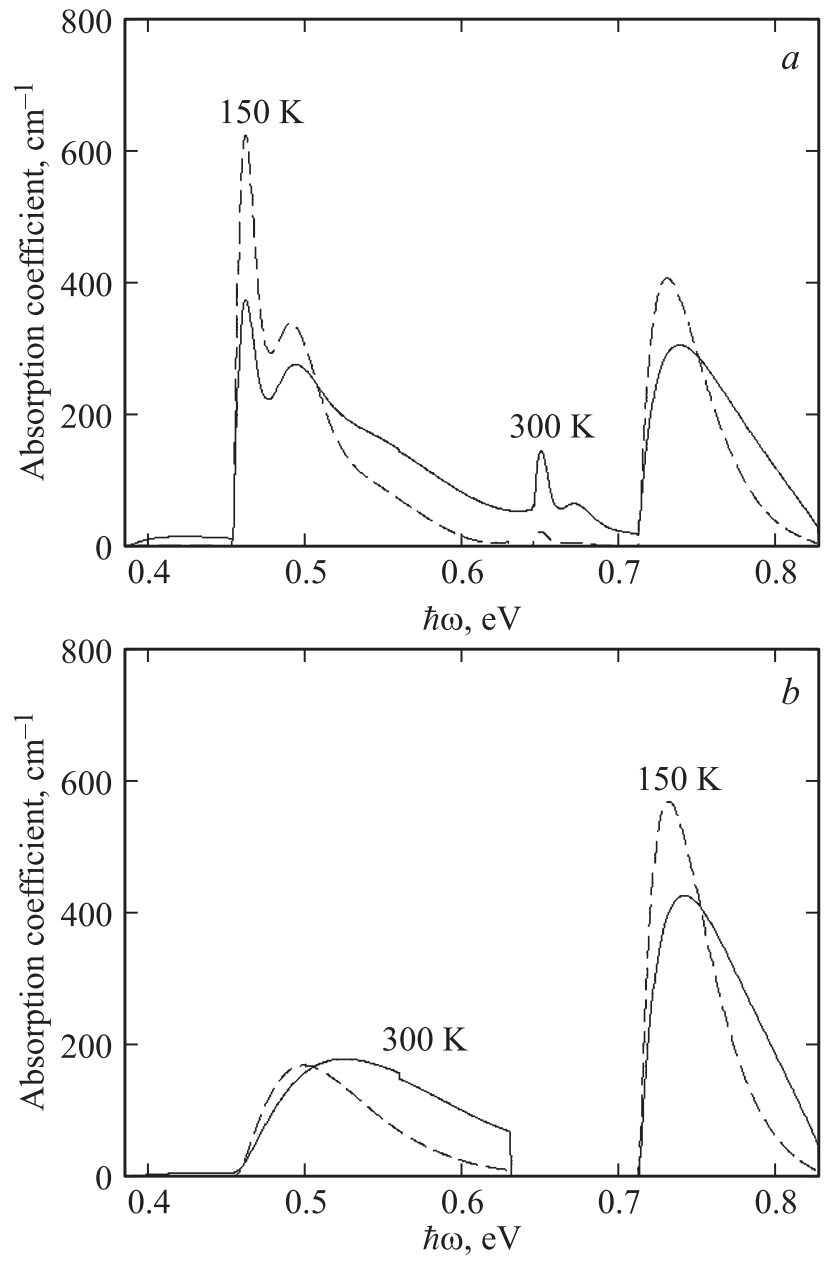

Рис. 3. Зависимости коэффициентов поглощения для переходов на дискретные уровни спин-отщепленных дырок $\alpha_{\|}^{d}(a)$ и $\alpha_{\perp}^{d}(b)$ от частоты падающего излучения в гетероструктуре $\mathrm{AlSb} / \mathrm{InAs}_{0.84} \mathrm{Sb}_{0.16} / \mathrm{AlSb}$ при ширине квантовой ямы $a=40 \AA$ и концентрации дырок $p=10^{12} \mathrm{~cm}^{-2}$ : расчет для температур 300 и $150 \mathrm{~K}$.

спектра so-дырок,

$$
\alpha_{\perp}^{d} \propto \frac{p\left[\omega-\omega_{\mathrm{th}}^{d}\left(k_{h}, k\right)\right]^{2}}{T},
$$

a $\alpha_{\|}^{d} \propto p / T$ (т.е. меняется на пороге скачком). Для переходов, происходящих с основного уровня тяжелых дырок на нечетные ветви so-дырок,

$$
\alpha_{p}^{d} \propto \frac{p\left[\omega-\omega_{\mathrm{th}}^{d}\left(k_{h}, k\right)\right]}{T} .
$$

В случае статистики Больцмана описанное поведение $\alpha_{p}^{d}$ должно наблюдаться приблизительно до частот

$$
\omega_{\mathrm{th}}^{d}\left(k_{h}, k\right)+\frac{m_{h} T}{m_{r}^{d}(k) \hbar} .
$$

Стоит отметить, что многие различия в поведении $\alpha_{\perp}^{d}$ и $\alpha_{\|}^{d}$ связаны с тем, что некоторые интегралы перекрытия $I_{i}^{j}(18)-(21)$ пропорциональны $q$, а другие пропорциональны $k_{h}$.
При значениях $q \gg q_{T}$ коэффициент поглощения

$$
\alpha_{p}^{d} \propto\left(\frac{p}{T}\right) \exp \left[-\frac{\hbar\left(\omega-\omega_{\mathrm{th}}^{e}\right)}{T}\right]
$$

так как его поведение в этой частотной области в основном определяется экспоненциально убывающей заселенностью состояний $f\left(E_{h}\right)$.

Представляет интерес температурная зависимость максимумов $\alpha_{\perp}^{d}(\omega)$ и $\alpha_{\|}^{d}(\omega)$. Сначала рассмотрим переходы, происходящие с основного уровня тяжелых дырок на уровни so-дырок с такой же четностью. Максимум $\alpha_{\|}^{d}(\omega)$ достигается при $\omega=\omega_{\text {th }}^{d}$ (мы по-прежнему ограничиваемся рассмотрением только первых членов в разложении $\alpha_{\|}^{d}(\omega)$ по степеням $\left.\omega-\omega_{\mathrm{th}}^{d}\right)$; при этой частоте $\alpha_{\|}^{d}(\omega) \propto 1 / T$.

Считая

$$
\alpha_{\perp}^{d} \propto \frac{p\left[\omega-\omega_{\mathrm{th}}^{d}\left(k_{h}, k\right)\right]^{2}}{T} \exp \left[-\frac{\hbar\left(\omega-\omega_{\mathrm{th}}^{d}\right)}{T}\right],
$$

получим, что максимум $\alpha_{\perp}^{d}(\omega)$ достигается при частоте, равной $\omega_{\text {th }}^{d}+2 T / \hbar$, а его величина $\propto T$. Для всех остальных переходов с основного уровня частота максимума увеличивается с ростом температуры, $\omega_{m}^{d}=\omega_{\mathrm{th}}^{d}+2 T / \hbar$, а его величина от температуры не зависит.

На рис. 3, $a$ и $b$ изображены частотные зависимости $\alpha_{\perp}^{d}$ и $\alpha_{\|}^{d}$ при различных температурах в гетероструктуре $\mathrm{AlSb} / \mathrm{InAs}_{0.84} \mathrm{Sb}_{0.16} / \mathrm{AlSb}$. Видно, что при $T=150 \mathrm{~K}$ наблюдаются более резкие пики с большей амплитудой, чем при $T=300 \mathrm{~K}$. Также видно, что при повышении температуры энергия перехода, соответствующая пикам поглощения, увеличивается.

На рис. 4, $a$ и $b$ изображены зависимости $\alpha_{\perp}^{d}$ и $\alpha_{\|}^{d}$ в гетероструктуре $\mathrm{AlSb} / \mathrm{InAs}_{0.84} \mathrm{Sb}_{0.16} / \mathrm{AlSb}$ для различных

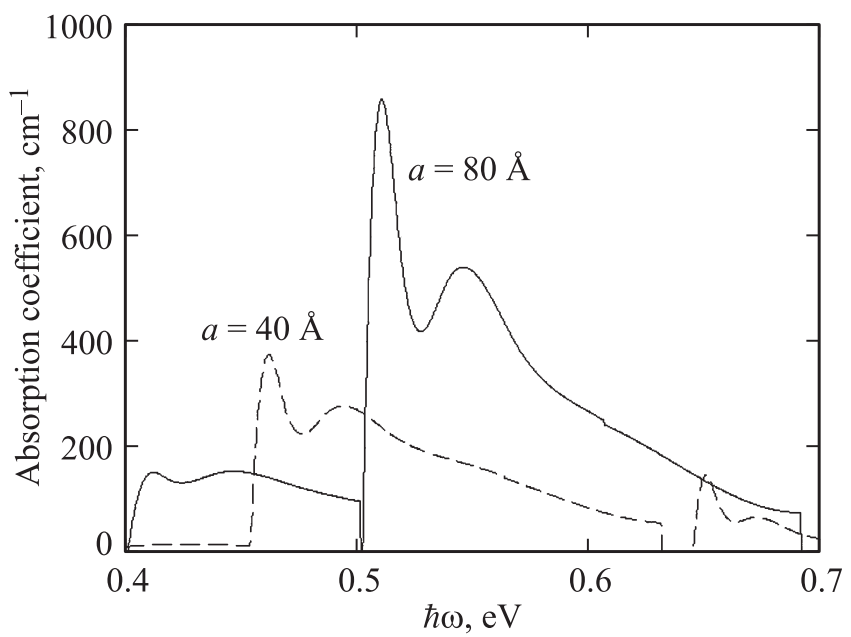

Рис. 4. Зависимости коэффициента поглощения для переходов на дискретные уровни спин-отщепленных дырок $\alpha_{\|}^{d}$ от частоты падающего излучения для различных значений ширины квантовой ямы в гетероструктуре $\mathrm{AlSb} / \mathrm{InAs}_{0.84} \mathrm{Sb}_{0.16} / \mathrm{AlSb}$ при температуре $T=300 \mathrm{~K}$ и концентрации дырок $p=10^{12} \mathrm{~cm}^{-2}$. 

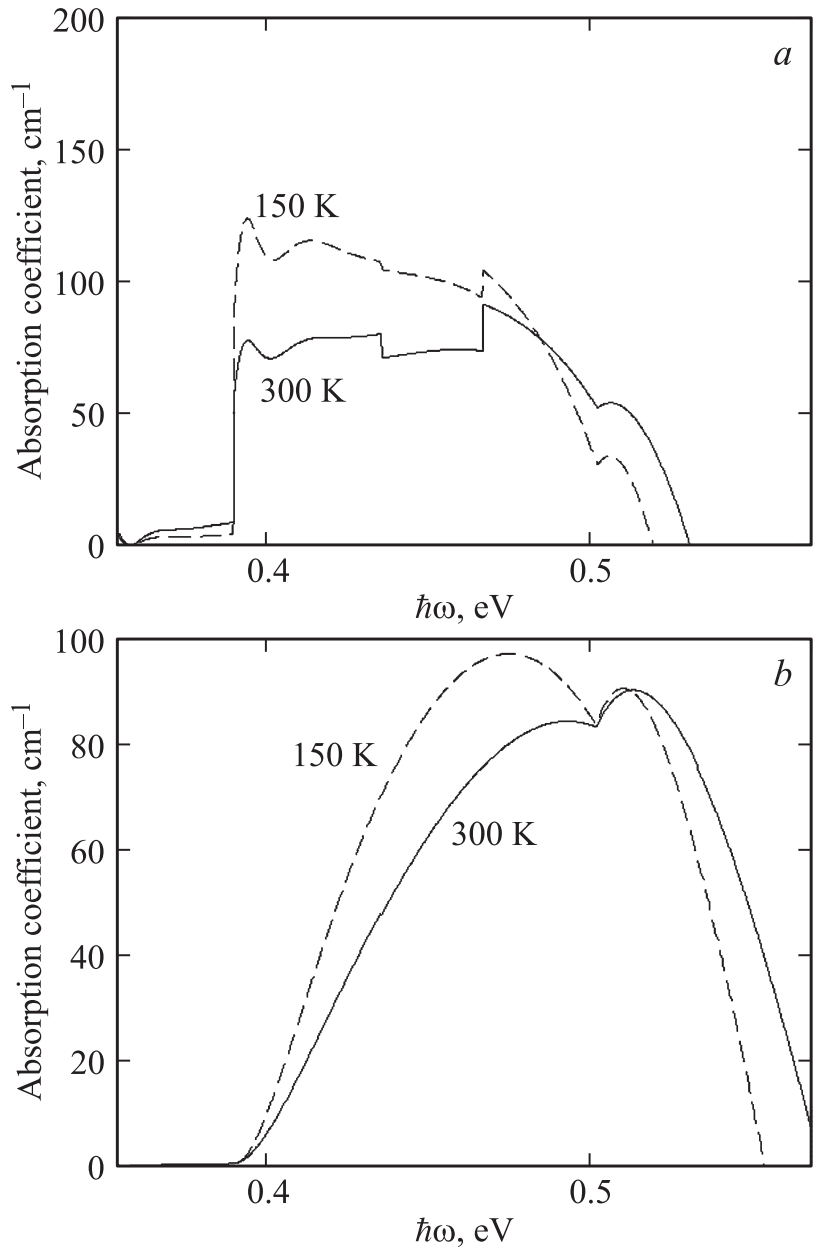

Рис. 5. Зависимости коэффициентов поглощения для переходов на дискретные уровни спин-отщепленных дырок $\alpha_{\|}^{d}(a)$ и $\alpha_{\perp}^{d}(b)$ от частоты падающего излучения в гетероструктуре InGaAsP/InP при ширине квантовой ямы $a=80 \AA$ и концентрации дырок $p=10^{12} \mathrm{~cm}^{-2}$ : расчет для температур 300 и $150 \mathrm{~K}$.

значений ширины квантовой ямы $a$. Видно, что при увеличении ширины квантовой ямы уменьшается интенсивность переходов с основного уровня тяжелых дырок, однако резко увеличивается интенсивность переходов с первого возбужденного уровня, так как его заселенность уже становится весьма значительной, а матричный элемент перехода в несколько раз больше в основном за счет величины $k_{h}^{2}$.

На рис. 5, $a$ и $b$ изображены частотные зависимости $\alpha_{\perp}^{d}$ и $\alpha_{\|}^{d}$ при различных температурах в гетероструктуре InGaAsP/InP. Видно, что значение коэффициента поглощения для данной гетероструктуры будет меньше, чем для гетероструктуры $\mathrm{AlSb} / \mathrm{InAs}_{0.84} \mathrm{Sb}_{0.16} / \mathrm{AlSb}$. Это происходит вследствие того, что $\alpha_{p}^{d} \propto S_{d}^{2} \propto\left(E_{g}+\Delta\right)^{-2}$, и для более узкозонных полупроводников внутризонное поглощение будет сильнее. Также отчетливо видно отсутствие для $\alpha_{\|}^{d}$ пика, связанного с переходом между первыми возбужденными подзонами тяжелых и спинотщепленных дырок. Помимо вышеуказанной причины, справедливой для всех переходов, в данном случае существенную роль играет то, что в гетероструктуре InGaAsP/InP значительно больше расстояние между подзонами размерного квантования тяжелых дырок и при данном значении температуры концентрация тяжелых дырок на первом возбужденном уровне мала.

В заключение сравним $\alpha_{\perp}^{d}(\omega)$ и $\alpha_{\|}^{d}(\omega)$ по величине. Как следует из изложенного выше в настощем разделе, при малых температурах излучение, поляризованное в плоскости квантовой ямы, поглощается эффективнее, чем излучение поперечной поляризации. Это объясняется тем, что в выражения (19), (20) для интегралов перекрытия $I_{y}^{d}$ и $I_{z}^{d}$ входит множителем либо $q$, либо $k_{h}$, а в выражения для $I_{x}^{d}$ входит множителем именно $q$, поэтому при увеличении $q$ преобладание $\alpha_{\|}^{d}(\omega)$ будет становиться менее заметным. В результате $\alpha_{\perp}^{c}(\omega)$ оказывается существенно меньшим, чем $\alpha_{\|}^{c}(\omega)$, причем преобладание $\alpha_{\|}^{c}(\omega)$ над $\alpha_{\perp}^{c}(\omega)$ довольно четко выражено даже при больших значениях температуры.
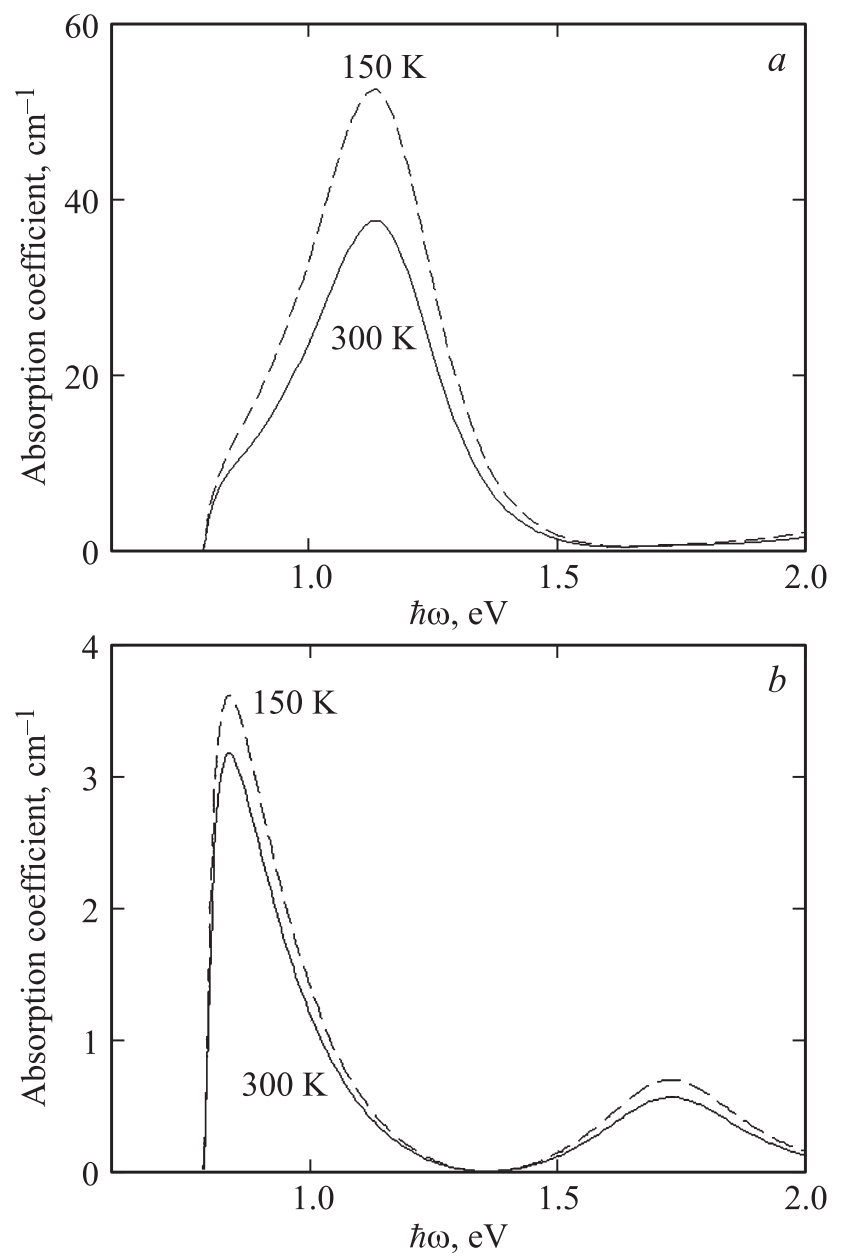

Рис. 6. Зависимости коэффициентов поглощения для переходов в непрерывный спектр спин-отщепленных дырок $\alpha_{\|}^{c}(a)$ и $\alpha_{\perp}^{c}(b)$ от частоты падающего излучения для различных температур в гетероструктуре $\mathrm{AlSb} / \mathrm{InAs}_{0.84} \mathrm{Sb}_{0.16} / \mathrm{AlSb}$ при ширине квантовой ямы $a=40 \AA$ и концентрации дырок $p=10^{12} \mathrm{~cm}^{-2}$ : расчет для температур 300 и $150 \mathrm{~K}$. 

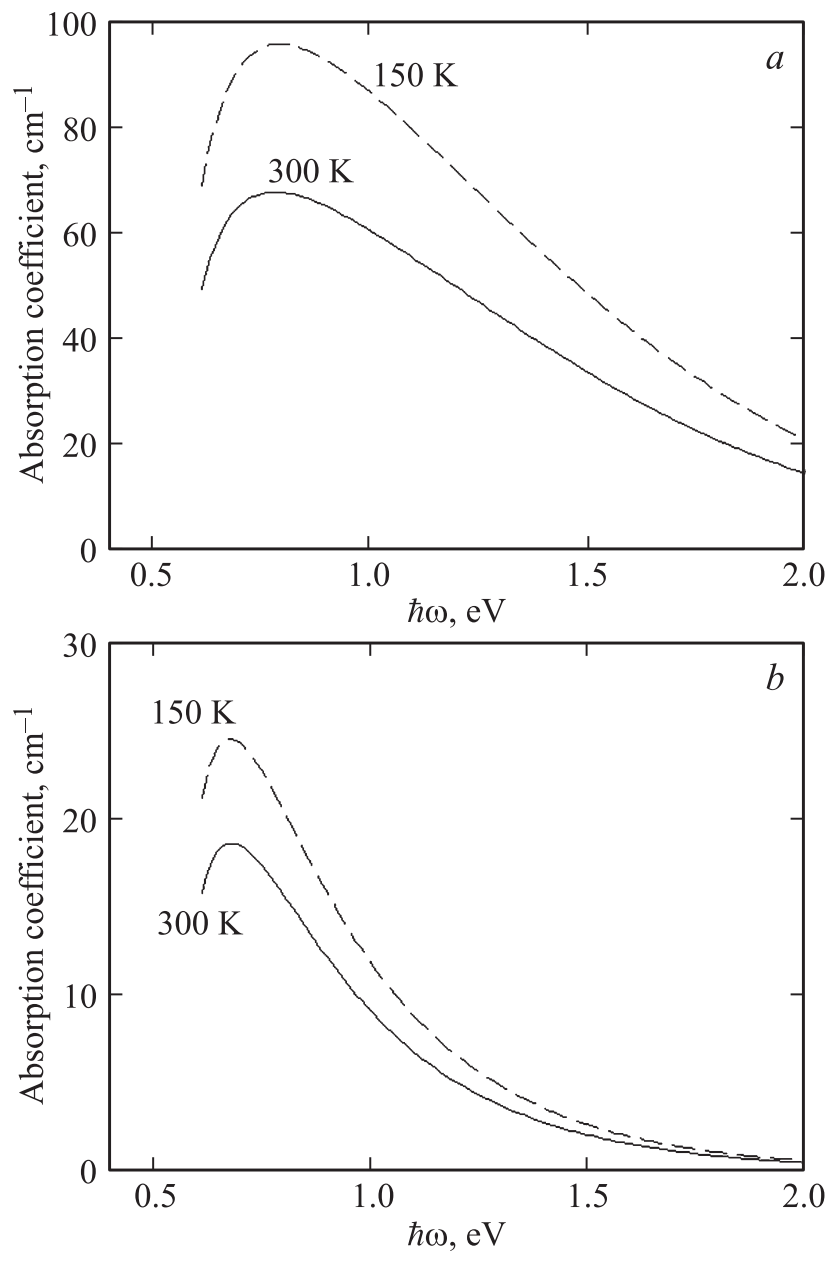

Рис. 7. Зависимости коэффициентов поглощения для переходов в непрерывный спектр спин-отщепленных дырок $\alpha_{\|}^{c}(a)$ и $\alpha_{\perp}^{c}(b)$ от частоты падающего излучения для различных температур в гетероструктуре InGaAsP/InP при ширине квантовой ямы $a=40 \AA$ и концентрации дырок $p=10^{12} \mathrm{~cm}^{-2}$ : расчет для температур 300 и $150 \mathrm{~K}$.

\section{2. Коэффициент поглощения, обусловленный переходами в непрерывный спектр so-дырок}

Коэффициент поглощения с переходами непрерывный спектр, $\alpha_{p}^{c}$, согласно (49) и (42), равен

$$
\alpha_{p}^{c}=\frac{\gamma^{2} e^{2}}{2 \pi \hbar^{2} c} \frac{m_{h}}{n \omega a} \sum_{k_{h}} \int_{E_{h}^{\min }}^{E_{h}^{\max }} \frac{\sigma_{p}^{c} f\left(E_{h}\right)}{k\left|E^{\prime}\left(K^{2}\right)\right|} d E_{h} .
$$

Суммирование по $k_{h}$ в (51) можно для облегчения анализа опустить так же, как мы это сделали в разд. 6.1. В случае статистики Больцмана формула (51) при частотах, близких к $\omega_{\mathrm{th}}^{c}\left(k_{h}, k\right)$, приводит к зависимостям $\alpha_{\perp}^{c}(\omega)$ и $\alpha_{\|}^{c}(\omega)$ степенно́го характера. В частности, если частота $\omega$ удовлетворяет неравенству

$$
\hbar \omega-\hbar \omega_{\mathrm{th}}^{c}\left(k_{h}, k\right) \ll \frac{m_{h} T}{\tilde{m}_{r}},
$$

$q$ оказывается меньшим, чем $q_{T}$, а коэффициенты поглощения растут с удалением от порога по законам

$$
\alpha_{\perp}^{c} \propto \frac{p\left[\omega-\omega_{\mathrm{th}}^{d}\left(k_{h}, k\right)\right]^{2}}{T}, \quad \alpha_{\|}^{c} \propto \frac{p\left[\omega-\omega_{\mathrm{th}}^{d}\left(k_{h}, k\right)\right]}{T} .
$$

На рис. $6, a$ и $b$ изображены частотные зависимости $\alpha_{\perp}^{c}$ и $\alpha_{\|}^{c}$ при различных температурах в гетероструктуре $\mathrm{AlSb} / \mathrm{InAs}_{0.84} \mathrm{Sb}_{0.16} / \mathrm{AlSb}$. Ход кривых соответствует зависимостям (52), однако также накладывается периодическая составляющая, так как коэффициенты $C_{1}$ и $C_{2}$ зависят от $k$ периодически, посредством множителей $\cos ^{2}(k a / 2)$ и $\sin ^{2}(k a / 2)$. Разный вид зависимостей обусловлен тем, что величина $\alpha_{\perp}^{c}$ определяется главным образом коэффициентом $C_{1}$, а величина $\alpha_{\|}^{c}-$ коэффициентом $C_{2}$.

На рис. 7, $a$ и $b$ изображены частотные зависимости $\alpha_{\|}^{c}$ и $\alpha_{\perp}^{c}$ при различных температурах в гетероструктуре InGaAsP/InP. Стоит отметить, что для переходов в непрерывный спектр значение коэффициента поглощения для данной гетероструктуры будет выше, чем для гетероструктуры $\mathrm{AlSb} / \mathrm{InAs}_{0.84} \mathrm{Sb}_{0.16} / \mathrm{AlSb}$, вследствие того, что влияние множителя $S_{c}^{2}$ компенсирует множитель $\tilde{N}_{c}$, входящий в знаменатель выражений для $C_{1}^{2}$ и $C_{2}^{2}$, и в этом случае основное влияние будет оказывать множитель $\hbar \omega$ в знаменателе выражения (51).

Отметим, что в гетероструктуре $\mathrm{AlSb} / \mathrm{InAs}_{0.84} \mathrm{Sb}_{0.16} /$ $\mathrm{AlSb}$ коэффициент поглощения в дискретный спектр существенно больше коэффициента поглощения в непрерывный спектр, а для InGaAsP/InP значения обоих коэффициентов сравнимы по величине.

\section{7. Заключение}

В работе выполнен микроскопический анализ механизма внутризонного поглощения излучения дырками с переходом в so-зону для квантовых ям на основе полупроводников $\mathrm{A}^{\mathrm{III}} \mathrm{B}^{\mathrm{V}}$. Отдельно рассмотрены случаи поперечной и продольной поляризаций падающего излучения. Анализ выполнен для случаев перехода в дискретный и в непрерывный спектр so-дырок. Показано, что такой механизм внутризонного поглощения может быть основным механизмом внутренних оптических потерь для полупроводниковых лазеров на квантовых ямах.

Следующий важный результат заключается в том, что спектр so-дырок в квантовых ямах существенно непараболичен (рис. 2). В работе [10] был вычислен внутризонный коэффициент поглощения дырками в $s o$-подзоне для однородных полупроводников и показано, что параболическое приближение дает в 2 раза больший коэффициент поглощения по сравнению с непараболическим случаем. Влияние непараболичности спектра so-дырок на коэффициент поглощения является более сильным в квантовых ямах, чем в однородном полупроводнике [10].

В квантовых ямах поглощение, происходящее с переходом в дискретный спектр so-дырок, является более сильным, чем поглощение, происходящее с переходом 
в непрерывный спектр. Частотная зависимость коэффициента поглощения, обусловленного переходами в дискретный спектр so-дырок, имеет ярко выраженные максимумы, положение и величина которых зависит от температуры. С ростом температуры пик смещается в сторону бо́льших энергий, а пиковое значение коэффициента поглощения убывает.

Значение коэффициента поглощения при переходах в дискретный спектр убывает при увеличении значения энергии запрещенной зоны материала. Для переходов в непрерывный спектр ярко выраженной зависимости от ширины запрещенной зоны не наблюдается. В этом случае более значительную роль играет обратно пропорциональная зависимость коэффициента поглощения от энергии перехода.

Также одним из интересных результатов работы является вывод о том, что поглощение излучения, поляризованного в плоскости квантовой ямы, преобладает над поглощением излучения поперечной поляризации.

Работа выполнена при поддержке Министерства образования и науки РФ в рамках Федеральной целевой программы „Исследования и разработки по приоритетным направлениям развития научно-технологического комплекса России на 2014-2020 г.“, шифр 2015-14582-0038, соглашение № 14.581.21.0013 от 4 августа 2015 г., уникальный идентификатор RFMEFI58115X0013.

\section{Приложение}

\section{Тяжелые дырки}

Выпишем в явном виде волновые функции тяжелых дырок $[16,17]$. Их $s$-компонента тождественно равна нулю. Для $v=1$ в области квантовой ямы, $-a / 2<x<a / 2$

$$
\begin{aligned}
\Psi_{h, p}= & H_{1}\left(\begin{array}{c}
q \cos k_{h} x \xi \\
-i k_{h} \sin k_{h} x \xi \\
-k_{h} \sin k_{h} x \xi+q \cos k_{h} x \eta
\end{array}\right) \\
& +H_{2}\left(\begin{array}{c}
q \sin k_{h} x \eta \\
i k_{h} \cos k_{h} x \eta \\
-k_{h} \cos k_{h} x \xi-q \sin k_{h} x \eta
\end{array}\right) .
\end{aligned}
$$

Здесь $q, k_{h}-x$ - и $y$-компоненты квазиимпульса тяжелых дырок, $H_{1}$ и $H_{2}$ - нормировочные коэффициенты.

В области барьера, $x>a / 2$, волновые функции связанных дырок экспоненциально затухают при удалении от интерфейса:

$$
\begin{aligned}
\Psi_{h, p}= & \tilde{H}_{1}\left(\begin{array}{c}
q \xi \\
-i \kappa_{h} \xi \\
-\kappa_{h} \xi+q \eta
\end{array}\right) e^{-\kappa_{h}(x-a / 2)} \\
& +\tilde{H}_{2}\left(\begin{array}{c}
q \eta \\
-i \kappa_{h} \eta \\
-q \xi+\kappa_{h} \eta
\end{array}\right) e^{-\kappa_{h}(x-a / 2)} .
\end{aligned}
$$

Здесь $\kappa_{h}-$ модуль $x$-компоненты квазиимпульса тяжелой дырки под барьером, а $\tilde{H}_{1}$ и $\tilde{H}_{2}-$ нормировочные коэффициенты.
Для области $x<-a / 2$ волновые функции могут быть получены из (П.2) с учетом соотношения (7). Переход к функциям другой симметрии $(v=-1)$ в (П.1), (П.2) может быть осуществлен путем формальной замены $\xi \leftrightarrow \eta$.

Строго говоря, волновые функции дырок в квантовой яме являются суперпозицией трех ветвей валентной зоны: тяжелых, легких и so-дырок. При энергиях, близких к $\delta$, ветвь спин-отщепленных дырок экспоненциально затухает при удалении от интерфейса с показателем $\kappa=\sqrt{4 m_{h} \Delta / 3 \hbar^{2}}$. Поэтому эта ветвь главным образом влияет на значение производной волновой функции вблизи гетерограницы, а ее влияние на значение самой волновой функции пренебрежимо мало. Таким образом, получается, что вблизи потолка валентной зоны волновую функцию можно искать в виде суперпозиции ветвей тяжелых и легких дырок. В случае использования приближений $\gamma=$ const, $\gamma_{1}=$ const, $\gamma_{2}=$ const и $m_{h} \gg m_{l}$ получим, что легкие и тяжелые дырки не смешиваются $[16,17]$. Спектр тяжелых дырок определяется уравнениями

$$
\begin{gathered}
\tan \frac{k_{h} a}{2}=\frac{\kappa_{h}}{k_{h}} \text { - для четных состояний, } \\
\cot \frac{k_{h} a}{2}=-\frac{\kappa_{h}}{k_{h}}-\text { для нечетных состояний. }
\end{gathered}
$$

Под четностью состояния тяжелой дырки мы понимаем четность $\Psi_{h, x}$. Используя (П.3), (П.4) можно определить константы нормировки $H_{1}$ и $H_{2}$ в (П.1):

$$
\begin{aligned}
H_{1}= & {\left[a\left(k_{h}^{2}+q^{2}\right)+\frac{2 q^{2}}{\kappa_{h}}\right]^{-1 / 2}, \quad H_{2}=0 } \\
& \text { - для четных состояний, } \\
H_{1}= & 0, \quad H_{2}=\left[a\left(k_{h}^{2}+q^{2}\right)+\frac{2 q^{2}}{\kappa_{h}}\right]^{-1 / 2} \\
& \text { - для нечетных состояний. }
\end{aligned}
$$

\section{Список литературы}

[1] G.P. Agrawal, N.K. Dutta. Long Wavelength Semiconductor Lasers (Van Nostrand Reinhold Co., N.Y., 1993).

[2] P.S. Zory. Quantum Well Lasers (Academic Press, 1993).

[3] E. Rosencher, B. Levine. Intersubband Transitions in Quantum Wells (Plenum, N.Y., 1992).

[4] А.Я. Шик. ФТП, 22 (10), 1843 (1988).

[5] W.J. Zawadski. J. Phys. C, 16, 229 (1983).

[6] А.Г. Петров, А.Я. Шик. ФТП, 28 (12), 2185 (1994).

[7] J.R. Hoff, M. Razeghi, G.J. Brown. Phys. Rev. B, 54 (15), 10733 (1996).

[8] F. Szmulowicz, G.J. Brown. Phys. Rev. B, 51 (19), 13203 (1995).

[9] E.O. Kane. J. Phys. Chem. Sol., 1, 249 (1957).

[10] N.A. Gun'ko, V.B. Khalfin, Z.N. Sokolova, G.G. Zegrya. J. Appl. Phys., 84 (1), 547 (1998). 
[11] G.G. Zegrya. In: Antimonide-Related Strained-Layer Heterostructures, ed. by M.O. Manasreh (Gordon and Breach Science Publishers, Amsterdam, 1997) p. 273.

[12] Л.В. Данилов, Г.Г. Зегря. ФТП, 42 (5), 573 (2007).

[13] Н.В. Павлов, Г.Г. Зегря. ФТП, 48 (9), 1217 (2014).

[14] Г.Г. Зегря, Н.А. Пихтин, Г.В. Скрынников, С.О. Слипченко, И.С. Тарасов. ФТП, 35 (8), 1001 (2000).

[15] Р.А. Сурис. ФТП, 20 (11), 2008 (1986).

[16] Г.Г. Зегря, А.С. Полковников. ЖЭТФ, 113 (4), 1491 (1998).

[17] A.S. Polkovnikov, G.G. Zegrya. Phys. Rev. B, 58 (7), 4039 (1998).

[18] M.G. Burt. J. Phys.: Condens. Matter, 4, 6651 (1992).

[19] А.И. Ансельм. Введение в теорию полупроводников (Наука, М., 1978).

Редактор Л.В. Шаронова

\section{Intraband light absorption by holes in InAsSb/AISb and InGaAsP/InP quantum wells}

N.V. Pavlov' ${ }^{1}$, G.G. Zegrya ${ }^{1,2}$, A.G. Zegrya ${ }^{1}$, V.E. Bugrov ${ }^{2}$

${ }^{1}$ loffe Institute, 194021 St. Petersburg, Russia

${ }^{2}$ ITMO University,

197101 St. Petersburg, Russia

Abstract A microscopic analysis of intraband light absorption by holes with their transition to a spin-orbit split-off band for InAsSb/AlSb and InGaAsP/InP quantum wells was performed within the framework of the four-band Kane model. The calculation is performed for two polarizations of the incident radiation: along the crystal growth axis and in the plane of the quantum well. It is shown that the absorption with the transition to the discrete spectrum of spin-split holes will be more intensive than that to the continuous spectrum. A detailed analysis of the intraband absorption coefficient dependence on the temperature, on the hole concentration, and on the quantum well width is produced. It is shown that the intraband light absorption can be the main mechanism of internal radiation losses for quantum well lasers. 